Gabriele Zini - Paolo Tartarini

\title{
Solar Hydrogen Energy Systems
}

Science and Technology for the Hydrogen Economy

照 Springer 


\section{Contents}

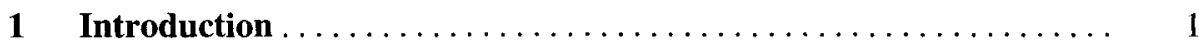

1.1 The Current Situation. . . . . . . . . . . . . . . . . . . 1

1.2 The Peak Oil Theory $\ldots \ldots \ldots \ldots \ldots \ldots \ldots \ldots \ldots \ldots \ldots \ldots$

1.3 Forms of Energy Sources and Environmental Impact ......... 4

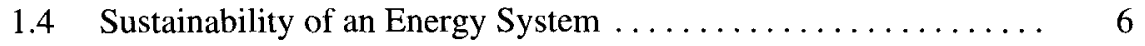

1.5 A Hydrogen New Energy System.................. 7

1.6 Scenarios for the Future . . . . . . . . . . . . . . . . . . . 7

1.7 Alternatives to Hydrogen $\ldots \ldots \ldots \ldots \ldots \ldots \ldots \ldots \ldots \ldots$

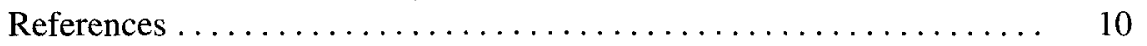

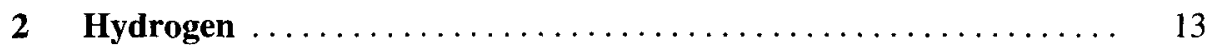

2.1 Hydrogen as Energy Carrier . . . . . . . . . . . . . . 13

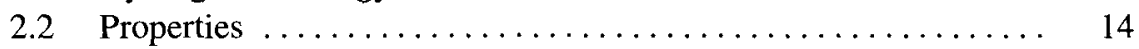

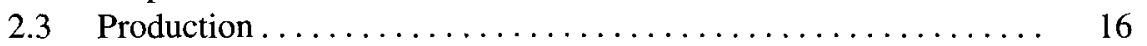

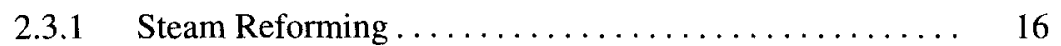

2.3.2 Solid Fuel Gasification $\ldots \ldots \ldots \ldots \ldots \ldots \ldots \ldots \ldots \ldots \ldots$

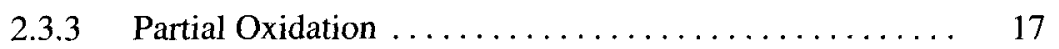

2.3 .4 Water Electrolysis . . . . . . . . . . . . . . . . 18

2.3.5 Thermo-Cracking $\ldots \ldots \ldots \ldots \ldots \ldots \ldots \ldots \ldots \ldots \ldots, 18$

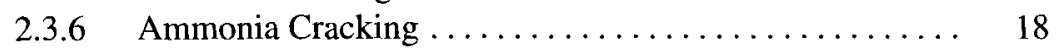

2.3.7 Other Systems: Photochemical, Photobiological,

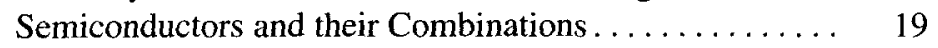

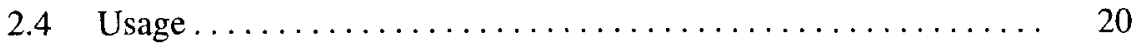

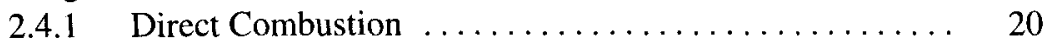

2.4.2 Catalytic Combustion .................. 23

2.4.3 Direct Steam Production from Combustion ......... 23

$2.4 .4 \quad$ Fuel Cell ......................... 23

2.5 Degenerative Phenomena and Material Compatibility ....... 24

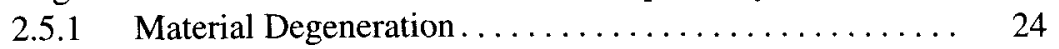

2.5 .2 Choice of Materials . . . . . . . . . . . . . . . . 25

2.6 Components: Pipes, Joints and Valves............... 26 
X Contents

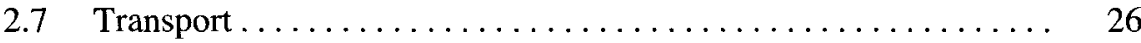

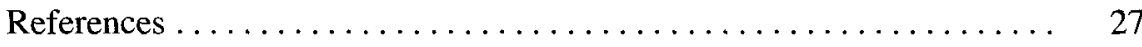

3 Electrolysis and Fuel Cells $\ldots \ldots \ldots \ldots \ldots \ldots \ldots \ldots \ldots \ldots \ldots \ldots$

3.1 Introduction. ............................... 29

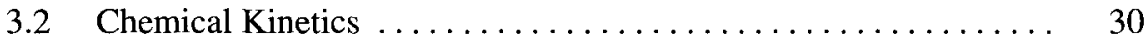

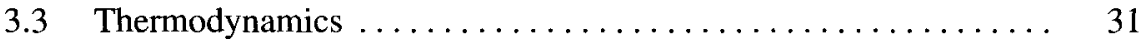

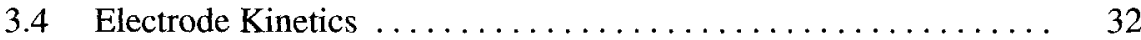

3.4.1 Activation Polarisation ................... 32

3.4 .2 Ohmic Polarisation ................... 33

3.4.3 Concentration Polarisation ................. 33

3.4.4 Reaction Polarisation ................... 34

3.4.5 Transfer Polarisation ....................... 34

3.4.6 Transport Phenomena ................... 34

3.4.7 Influence of Temperature and Pressure on Polarisation Losses ............................... 35

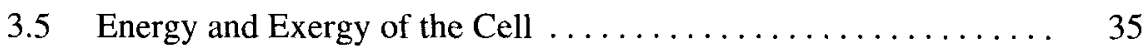

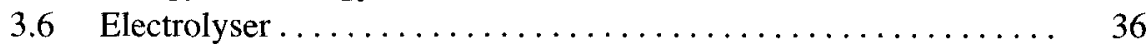

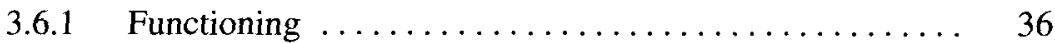

3.6 .2 Technology .......................... 37

3.6.2.1 Alkaline Electrolysers ............. 37

3.6.2.2 Solid Polymer (Polymeric Membrane)

Electrolysers................... 38

3.6.2.3 High-Temperature Electrolysers . . . . . . . 38

3.6.3 Thermodynamics ....................... 39

3.6.4 Mathematical Model ................... 40

3.6.5 Thermal Model ........................ 42

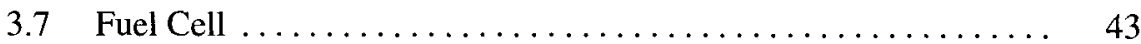

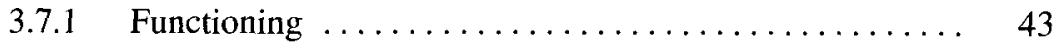

3.7.2 Technology ......................... 45

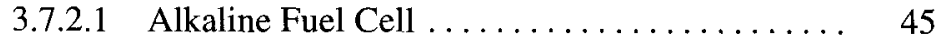

3.7.2.2 Phosphoric Acid Fuel Cell ............ 45

3.7.2.3 Polymeric Electrolyte Membrane Fuel Cell ... . 46

3.7.2.4 Molten Carbonate Fuel Cell ............. 46

3.7.2.5 Solid Oxide Fuel Cell ................ 47

3.7.3 Thermodynamics ...................... 48

3.7.4 Mathematical Model .................. 50

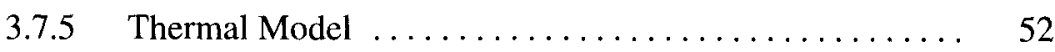

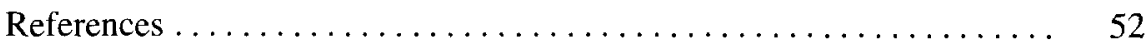

4 Solar Radiation and Photovoltaic Conversion $\ldots \ldots \ldots \ldots \ldots \ldots \ldots$

4.1 Solar Radiation . . . . . . . . . . . . . . . . . . . . 53

4.2 Photovoltaic Effect, Semiconductors and the p-n Junction ...... 55

4.3 Crystalline Silicon Photovoltaic Cells ................ 58

4.4 Other Cell Technologies $\ldots \ldots \ldots \ldots \ldots \ldots \ldots \ldots \ldots \ldots \ldots \ldots$ 
4.5 Conversion Losses ............................. 61

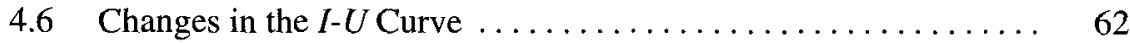

4.7 Photovoltaic Cells and Modules .................. 63

4.8 Types of Photovoltaic Plants . . . . . . . . . . . . . . . 65

4.9 Radiation on the Receiving Surface ................ 67

4.10 Determination of the Operating Point $\ldots \ldots \ldots \ldots \ldots \ldots \ldots$

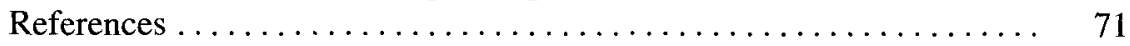

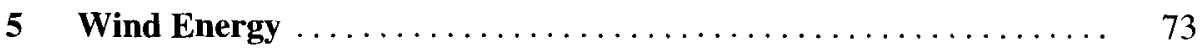

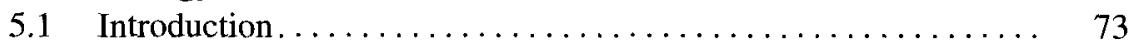

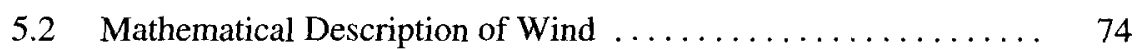

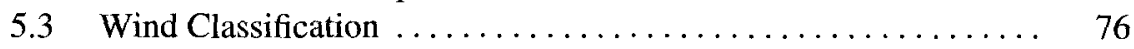

5.4 Mathematical Model of the Aerogenerator . . . . . . . . . . 77

5.5 Power Control and Design $\ldots \ldots \ldots \ldots \ldots \ldots \ldots \ldots \ldots \ldots \ldots$

5.6 Wind Turbine Rating $\ldots \ldots \ldots \ldots \ldots \ldots \ldots \ldots \ldots \ldots \ldots \ldots \ldots$

5.7 Electric Energy Conversion $\ldots \ldots \ldots \ldots \ldots \ldots \ldots \ldots \ldots \ldots \ldots$

5.8 Calculation Example $\ldots \ldots \ldots \ldots \ldots \ldots \ldots \ldots \ldots \ldots \ldots \ldots$

5.9 Environmental Impact $\ldots \ldots \ldots \ldots \ldots \ldots \ldots \ldots \ldots \ldots \ldots \ldots . \ldots \ldots$

References ............................. 89

6 Other Renewable Energy Sources for Hydrogen Production ...... 91

6.1 Solar Thermal Energy . . . . . . . . . . . . . . . . . 91

6.2 Hydroelectric Energy . . . . . . . . . . . . . . . . 93

6.3 Tidal, Wave and Ocean Thermal Energy Conversions.......... 93

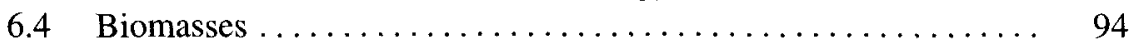

References ............................... 95

$7 \quad$ Hydrogen Storage $\ldots \ldots \ldots \ldots \ldots \ldots \ldots \ldots \ldots \ldots \ldots \ldots \ldots$

7.1 Issues of Hydrogen Storage . . . . . . . . . . . . . . 97

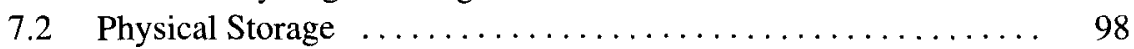

7.2.1 Compression Storage $\ldots \ldots \ldots \ldots \ldots \ldots \ldots \ldots \ldots$

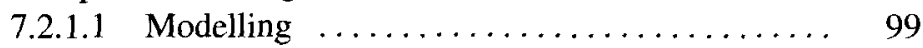

7.2.1.2 Dimensioning Example............. 101

7.2 .2 Liquefaction Storage $\ldots \ldots \ldots \ldots \ldots \ldots \ldots \ldots \ldots \ldots \ldots$

7.2.3 Glass or Plastic Containments ............. 103

7.3 Physical-Chemical Storage $\ldots \ldots \ldots \ldots \ldots \ldots \ldots \ldots \ldots \ldots \ldots . \ldots \ldots$

7.3.1 Physisorption .......................... 104

7.3.2 Empirical Models of Molecular Interactions ......... 105

7.3.3 Adsorption and Desorption Velocities ........... 107

7.3.4 Experimental Measurements of Adsorption and
Desorption $\ldots \ldots \ldots \ldots \ldots \ldots \ldots \ldots \ldots \ldots \ldots \ldots \ldots \ldots$

7.3.5 Adsorption Isotherms . . . . . . . . . . . . 109

7.3.6 Thermodynamics of Adsorption $\ldots \ldots \ldots \ldots \ldots \ldots \ldots 111$

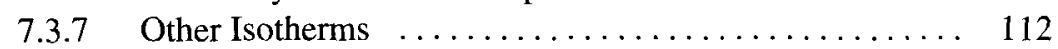

7.3.8 Classification of Isotherms $\ldots \ldots \ldots \ldots \ldots \ldots \ldots \ldots \ldots$ 
7.3.9 Carbon Materials for the Physisorption of Hydrogen ... 113

7.3.9.1 Nanotubes............................ 113

7.3.9.2 Activated Carbons ................ 114

7.3.10 Alternatives to Carbon Physisorption ............ 115

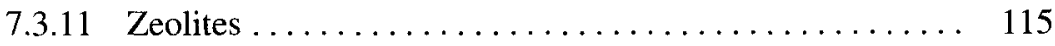

7.3 .12 Metallic Hydrides $\ldots \ldots \ldots \ldots \ldots \ldots \ldots \ldots \ldots \ldots \ldots$

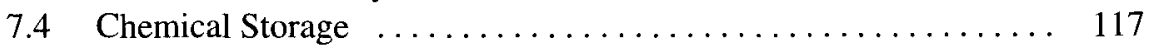

7.4 .1 Chemical Hydrides $\ldots \ldots \ldots \ldots \ldots \ldots \ldots \ldots \ldots \ldots . \ldots \ldots$

References ............................ 118

8 Other Electricity Storage Technologies $\ldots \ldots \ldots \ldots \ldots \ldots \ldots \ldots \ldots$

$8.1 \quad$ Introduction. . . . . . . . . . . . . . . . . . . . . . 121

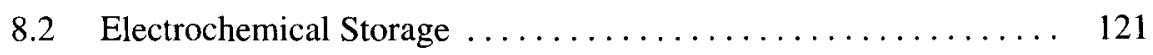

8.2.1 Valve Regulated Lead-Acid ................ 123

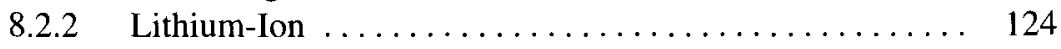

$8.2 .3 \quad$ Vanadium Redox ..................... 125

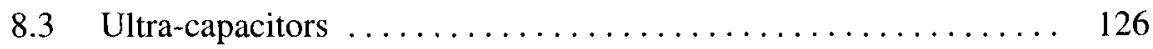

8.4 Compressed Air . ........................ 127

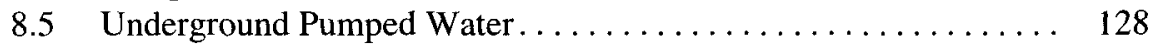

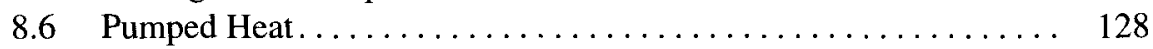

8.7 Natural Gas Production $\ldots \ldots \ldots \ldots \ldots \ldots \ldots \ldots \ldots \ldots \ldots \ldots \ldots \ldots$

8.8 Flywheels . . . . . . . . . . . . . . . . . . . . . . 129

8.9 Superconducting Magnetic Energy Storage $\ldots \ldots \ldots \ldots \ldots \ldots . \ldots 130$

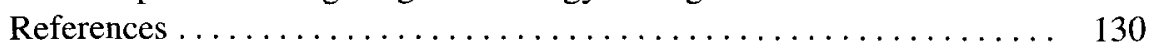

9 Study and Simulation of Solar Hydrogen Energy Systems . . . . . . 133

9.1 Solar Hydrogen Energy Systems . . . . . . . . . . . . . . . 133

9.2 Control Logic . . . . . . . . . . . . . . . . . . . 134

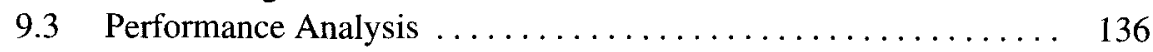

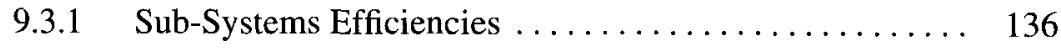

9.3.1.1 Photovoltaic Modules .............. 136

9.3.1.2 Aerogenerator ................ 137

9.3.1.3 Electrolyser .................. 137

9.3.1.4 Fuel Cell .................... 138

9.3.1.5 Compressor .................. 138

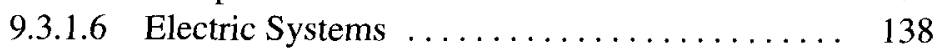

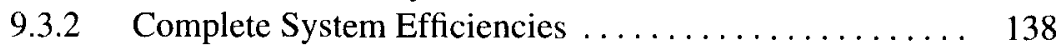

9.3.2.1 Hydrogen Production Efficiency .......... 139

9.3.2.2 Direct Route Efficiency............... 139

9.3.2.3 Hydrogen Loop Efficiency ............. 139

9.3.2.4 Complete System Efficiency ........... 140

9.4 Simulation with PV Conversion and Compression Storage... . . . 140

9.5 Simulation with PV Conversion and Activated-Carbon Storage... 147

9.6 Simulation with Wind Energy Conversion, Compression and Activated-Carbon Storage 
9.7 Notes on Exergy Analysis . . . . . . . . . . . . . . . . . 162

9.8 Remarks on the Simulation of Solar Hydrogen Energy Systems .. 162

References ................................. 163

10 Real-Life Implementations of Solar Hydrogen Energy Systems . . . . 167

10.1 Introduction. ................................. 167

10.2 The FIRST Project $\ldots \ldots \ldots \ldots \ldots \ldots \ldots \ldots \ldots \ldots \ldots \ldots \ldots \ldots \ldots$

10.3 The Schatz Solar Hydrogen Project . . . . . . . . . . . . . . . 169

10.4 The ENEA Project ................................ 170

10.5 The Zollbruck Full Domestic System $\ldots \ldots \ldots \ldots \ldots \ldots \ldots . \ldots 171$

10.6 The GlasHusEtt Project ............................. 172

10.7 The Trois Rivière Plant .......................... 172

10.8 The SWB Industrial Plant $\ldots \ldots \ldots \ldots \ldots \ldots \ldots \ldots \ldots \ldots \ldots$

10.9 The HaRI Project ............................. 174

10.10 Results from Real-Life Implementations . . . . . . . . . . . . . . 175

References ............................... 176

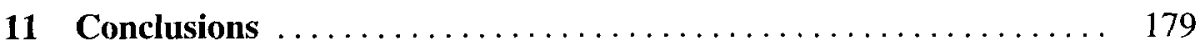

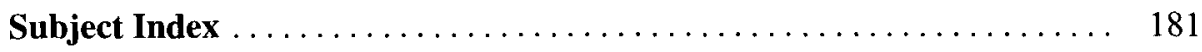

